\title{
Revisión
}

\section{Estado del arte del síndrome cardiorrenal, ventajas y limitaciones de las terapias conocidas}

\author{
María Carolina Manzur Barbur (DD $\square^{1}$, Ana Milena Mejía Sanjuanelo (D)1, \\ Marco Anaya Taboada ${ }^{2}{ }^{2}$, Juan Camilo García Domínguez (iD ${ }^{3}$ y Alejandra \\ Molano Triviño $\mathbb{D}^{4}$ \\ ${ }^{1}$ Departamento de Medicina Interna, Facultad de Medicina, Universidad del Norte, Barranquilla, Colombia. \\ ${ }^{2}$ Departamento de Nefrología, Universidad del Rosario, Bogotá, Colombia. \\ ${ }^{3}$ Departamento de Cardiología, Universidad del Bosque, Bogotá, Colombia. \\ ${ }^{4}$ Departamento de Nefrología, Universidad Javeriana, Bogotá, Colombia.
}

Cómo citar: Manzur-Barbur MC, Mejía-Sanjuanelo AM, Anaya-Taboada M, García- Domínguez JC, Molano-Triviño A. Estado del arte del síndrome cardiorrenal, ventajas y limitaciones de las terapias conocidas. Rev. Colomb. Nefrol. 2021 8(2), e517. https:/doi.org/10.22265/acnef.8.2.517

Recibido:

$16 / \mathrm{Sep} / 2020$

Aceptado:

09/Dic/2020

Publicado:

$23 / \mathrm{Feb} / 2021$

Resumen

Las interacciones entre las enfermedades cardiacas y las renales se han clasificado como síndromes cardiorrenales. La clasificación actual incluye cinco subtipos: insuficiencia cardiaca aguda que conduce a insuficiencia renal aguda (tipo 1), insuficiencia cardiaca crónica que conduce a insuficiencia renal (tipo 2), lesión renal aguda que conduce a insuficiencia cardiaca (tipo 3), enfermedad renal crónica que conduce a insuficiencia cardiaca (tipo 4) y afecciones sistémicas que conducen a insuficiencia cardiaca y renal (tipo 5) (tabla 1) [1,2].

En esta revisión discutimos la definición, la clasificación y la fisiopatología del síndrome cardiorrenal, enfocándonos en el manejo en fases agudas y su recuperación, al exponer la evidencia actualmente disponible de los diuréticos y la ultrafiltración, con el objetivo de intervenir de manera oportuna a nuestros pacientes al conocer las ventajas y las limitaciones de cada una de las estrategias de manejo en aras de reducir el riesgo de eventos clínicos, rehospitalización y muerte.

Palabras clave: síndrome cardiorrenal, falla cardiaca descompensada, diurético, resistencia al diurético, ultrafiltración, diálisis peritoneal.

$\checkmark$ Correspondencia: Universidad del Norte, calle 99 \#58-46, Barranquilla, Colombia. Correo electrónico: mbarbur@uninorte.edu.co 


\section{State of the art of cardiorenal syndrome, advantages and limitations of known therapies}

\section{Abstract}

Interactions between heart and kidney disease have been classified as cardiorenal syndromes. The current classification includes five subtypes, which are: acute heart failure leading to acute kidney failure (type 1), chronic heart failure leading to kidney failure (type 2), acute kidney injury leading to heart failure (type 3), chronic kidney disease leading to heart failure (type 4) and systemic conditions leading to heart and kidney failure (type 5) (table 1) [1,2].

In this review, we discuss the definition, classification, pathophysiology, focusing on acute phases treatment and its recovery, exposing the actual evidence for diuretics and ultrafiltration in order to intervene in a timely manner, pointing out the main advantages and limitations of each of the available strategies of treatment in order to reduce the risk of clinical events, re-hospitalization and death.

Keywords: cardiorrenal syndrome, decompensated heart failure, diuretic, diuretic resistance, ultrafiltration, peritoneal dialysis.

\section{Introducción}

El síndrome cardiorrenal (SCR) se caracteriza por una clínica variable, una fisiopatología compleja no dilucidada por completo, con una alta carga de morbimortalidad [1-3]. Existe un alto número de hospitalizaciones por SCR en Estados Unidos y en Europa, las cuales corresponden al $90 \%$ del millón de hospitalizaciones anuales por falla cardíaca descompensada, la mayoría de ellas por manifestaciones derivadas de la sobrecarga de líquidos $[1,2]$.

La terapia estándar para el manejo de la falla cardiaca descompensada consiste en diuréticos de asa y vasodilatadores [1,4]. El perfil de seguridad, eficacia y limitaciones derivadas, especialmente de los efectos adversos y la resistencia a los diuréticos que pueden presentar algunos pacientes, genera cierta preocupación y son motivo de investigación $[1,4]$.

El objetivo de esta revisión es hacer un repaso de los mecanismos fisiopatológicos implicados en los síndromes cardiorrenales, las opciones terapéuticas farmacológicas, las herramientas disponibles para delimitar la resistencia a los diuréticos y el papel de las terapias de ultrafiltración. 


\section{Definición}

El SCR es conocido como un espectro de los trastornos que involucran tanto el corazón como los riñones, en los que la disfunción aguda o crónica en un órgano puede inducir una disfunción aguda o crónica en el otro órgano [1,5-9].

El primer intento de definir formalmente el SCR provino del grupo de trabajo del National Heart, Lung, and Blood Institute de Estados Unidos en 2004, y fue conceptualizado formalmente como el resultado de interacciones entre los riñones y otros compartimentos circulatorios que aumentan el volumen circulante que exacerba los síntomas de insuficiencia cardiaca y la progresión de la enfermedad $[1,10,11]$.

\section{Clasificación}

La Acute Dialysis Quality Initiative describió un enfoque consensuado en 2008 al reconocer un espectro clínico más amplio que puede representar una desregulación cardiorrenal, según dos fenotipos principales: síndromes cardiorrenales y renocardiacos, basados en el compromiso primario del proceso de la enfermedad, los cuales fueron agrupados posteriormente en cinco subtipos: tabla $1[1,2,11,12]$.

\section{Fisiopatología}

La explicación convencional para el desarrollo del SCR en el contexto de trastorno cardiaco primario se basa en la incapacidad del corazón como bomba, para producir un adecuado flujo anterógrado que se traduce en hipoperfusión renal, lo cual conlleva a la activación del eje renina-angiotensina-aldosterona (RAA) del sistema nervioso simpático (SNS) y de la secreción de vasopresina, dando como resultado un aumento de la reabsorción tubular proximal de sodio y agua para mantener volúmenes plasmáticos efectivos, lo que eventualmente resulta en oliguria, mayor congestión, aumento de la precarga y empeoramiento de falla de bomba, tanto en falla cardiaca con fracción de eyección del ventrículo izquierdo (Fevi) como disminuida o preservada $[1,13,14]$.

A través del registro Adhere (Registro Nacional de Insuficiencia Cardiaca Descompensada Aguda) se demostró que la incidencia de aumento de la creatinina sérica fue similar entre los pacientes con insuficiencia cardiaca aguda, con función sistólica reducida y preservada [1,15]. 
Tabla 1. Clasificación del SCR basado en el consenso Acute Dialysis Quality Initiative

\begin{tabular}{|c|c|c|c|}
\hline Fenotipo & Nomenclatura & Descripción & Ejemplo \\
\hline SCR tipo 1 & SCR agudo & $\begin{array}{l}\text { Falla cardiaca aguda que } \\
\text { desencadena lesión renal } \\
\text { aguda (LRA) }\end{array}$ & $\begin{array}{l}\text { Síndrome coronario agudo } \\
\text { que desencadena choque } \\
\text { cardiogénico y LRA, falla } \\
\text { cardiaca aguda que } \\
\text { desencadena LRA }\end{array}$ \\
\hline SCR tipo 2 & SCR crónico & $\begin{array}{l}\text { Falla cardiaca crónica } \\
\text { que desencadena } \\
\text { enfermedad renal } \\
\text { crónica (ERC) }\end{array}$ & Falla cardiaca crónica \\
\hline SCR tipo 3 & $\begin{array}{l}\text { Síndrome renocardiaco } \\
\text { agudo }\end{array}$ & $\begin{array}{l}\text { Lesión renal aguda que } \\
\text { desencadena falla } \\
\text { cardiaca aguda }\end{array}$ & $\begin{array}{l}\text { Falla cardiaca en el } \\
\text { escenario de LRA por } \\
\text { sobrecarga hídrica, } \\
\text { alteraciones metabólicas por } \\
\text { uremia e inflamatorias }\end{array}$ \\
\hline SCR tipo 4 & $\begin{array}{l}\text { Síndrome renocardiaco } \\
\text { crónico }\end{array}$ & $\begin{array}{l}\text { ERC que desencadena } \\
\text { falla cardiaca crónica }\end{array}$ & $\begin{array}{l}\text { Hipertrofia ventricular } \\
\text { izquierda, falla cardiaca por } \\
\text { cardiomiopatía asociada a ERC }\end{array}$ \\
\hline SCR tipo 5 & $\begin{array}{l}\text { Síndrome cardiorenal } \\
\text { secundario }\end{array}$ & $\begin{array}{l}\text { Enfermedad sistémica } \\
\text { que desencadena falla } \\
\text { cardiaca y renal }\end{array}$ & Amiloidosis, sepsis, cirrosis \\
\hline
\end{tabular}

Fuente: adaptado con base en Rangaswami J, Bhalla V, Blair J, Chang T, Costa S, Lentine K, et al., $2019[1]$.

Los riñones tienen un circuito arterial de baja resistencia y reciben el $25 \%$ del gasto cardiaco, requiriendo un adecuado flujo sanguíneo renal venoso y arterial para mantener el filtrado glomerular [1]. En este contexto, las presiones venosas centrales elevadas resultan en hipertensión venosa renal, aumento de la resistencia renal y, en última instancia, disminución del flujo sanguíneo renal $[10,16]$.

Varias vías adicionales están involucradas en la lesión cardiaca o renal para el desarrollo del SCR, estas implican activación del SNS, inflamación crónica, desequilibrio en la proporción de especies reactivas de oxígeno/producción de óxido nítrico y activación persistente del eje renina angiotensina aldosterona $[1,5]$. La producción de citoquinas activas del SNS genera un aumento de las concentraciones séricas de noradrenalina, además se provoca la liberación de especies reactivas de oxígeno de los leucocitos y crea un ciclo vicioso por medio de los distintos mecanismos [5]. 
Se han evidenciado, principalmente implicadas como sustancias proinflamatorias en lesión renal aguda en modelos experimentales, varias moléculas inflamatorias como TNF- $\alpha$ (factor de necrosis tumoral- $\alpha$ ), IL-1 (interleucina-1) e IL-6 (interleucina-6), con efectos deletéreos directos también a nivel cardiaco $[1,17]$.

Como resultado de la inflamación y el estrés oxidativo se genera fibrosis, un marcador de irreversibilidad, y se cronifica la insuficiencia cardiaca y la lesión renal a enfermedad renal crónica, lo que ha llevado a algunos autores a etiquetarla como un mecanismo clave en la fisiopatología del SCR $[5,18]$. De igual manera, en la cardiomiopatía urémica (SCR tipo 4) hay hipertrofia del ventrículo izquierdo, lo que resulta en reducción en la densidad capilar e isquemia microvascular, las cuales se asocian con la progresión de la cardiopatía urémica [1,5]. Dichos mecanismos tienen una relación interdependiente entre la respuesta cardiaca y la renal $[5]$.

\section{Manejo farmacológico de la congestión en presencia de SCR}

\section{Terapias descongestivas}

Los objetivos del tratamiento farmacológico del SCR consisten en lograr una mejoría de los signos de congestión vascular y asegurar presiones adecuadas para garantizar la perfusión tisular [19-21]. Los diuréticos son la piedra angular del manejo descongestivo, se prescriben en aproximadamente el $90 \%$ de los pacientes con insuficiencia cardiaca descompensada con sobrecarga hídrica, por sus efectos en la disminución de la presión venosa central resultado de las pérdidas urinarias de sodio y agua $[1,5]$.

Los diuréticos de asa inhiben el cotransportador de $\mathrm{Na}+\mathrm{K}+2 \mathrm{Cl}$ - en la rama ascendente gruesa del asa de Henle [1]. Se prefieren respecto a los demás diuréticos debido a su menor incidencia de hipocalemia, hiponatremia y empeoramiento de la función renal [5]. No obstante, aunque su uso parece ser seguro y efectivo en la insuficiencia cardiaca descompensada, no se conoce con certeza si eleva los niveles de creatinina en individuos con una función renal basal más grave, por ejemplo, enfermedad renal crónica 4 o 5 [1].

Al respecto, el estudio DOSE (protocolo prospectivo, doble ciego y aleatorizado) evaluó las estrategias diuréticas con furosemida endovenosa administrada en bolo, dos veces al día o en infusión continua y comparando dosis bajas (dosis IV numéricamente equivalente a la dosis oral del paciente) y dosis altas (dosis IV, 2,5 veces equivalente a la dosis oral) [22]. En sus resultados no hubo diferencia significativa en la evaluación global de los síntomas o cambio en la creatinina sérica durante 72 h con administración diurética en bolo o infusión continua, 
o con una dosis baja versus una dosis alta; sin embargo, los pacientes asignados a la estrategia de dosis más altas experimentaron un incremento de creatinina transitoria [22].

El tratamiento con diuréticos, a diferencia de muchas otras terapias farmacológicas para la insuficiencia cardiaca, sigue siendo en gran medida empírico [1,19]. Las mejores prácticas clínicas basadas en la evidencia para el uso de diuréticos siguen siendo inciertas, ya que si bien proporcionan un rápido alivio de los síntomas, no han demostrado beneficio en desenlaces de mortalidad o de rehospitalización [1,19].

\section{Resistencia a los diuréticos}

Es ampliamente conocido que el uso recurrente de diuréticos en el SCR puede conllevar a la disminución de su eficacia, lo que se conoce como resistencia a los diuréticos [1]. Esto puede precipitarse con el uso de dosis sucesivas por medio de dos mecanismos: a corto plazo se genera el fenómeno de frenado cuando los diuréticos reducen el volumen de líquido extracelular, de manera que se activa el SNS y la producción de angiotensina II, lo que estimula la reabsorción de sodio tubular proximal y a largo plazo se relaciona con hipertrofia del túbulo contorneado distal (zona del cotransportador sodio/cloro sensible a tiazidas) y del túbulo colector, lo que conlleva al incremento de la reabsorción de sodio mediada por dichos segmentos de la nefrona $[1,19]$.

Si bien conocemos el término de resistencia al diurético, no está bien establecida una definición global, ni mucho menos una estrategia para diagnosticarla de manera certera. No obstante, la importancia clínica de la resistencia a los diuréticos se ve reforzada por investigaciones recientes, en las cuales se ha destacado la respuesta diurética como un fuerte predictor pronóstico al constituir un factor independiente de mayor mortalidad y rehospitalización por insuficiencia cardiaca, por lo cual se han propuesto varias alternativas para el manejo de la resistencia a los diuréticos [23]. A continuación, mencionaremos algunas alternativas de manejo:

1. Optimización de la farmacocinética de la terapia con diuréticos de asa al pasar de la administración oral a la intravenosa para evitar la absorción variable que ocurre en estados congestivos [23].

2. Bloqueo tubular: añadir diuréticos tiazídicos por su efecto diurético a nivel distal [24]. Las tiazidas siguen siendo el diurético de elección para combinar (bloquean entre 5 y $10 \%$ de la reabsorción de sodio filtrado en comparación al $25 \%$ de diuréticos de asa), contrarrestando la hipertrofia tubular distal compensatoria [15, 23, 24]. Los antagonistas de lso receptores de mineralocorticoides en dosis bajas, administrados a pacientes con insuficiencia cardiaca con FEVI disminuida, se asocian con una menor mortalidad, por 
lo que se encuentran incluidos en guías de manejo [15] y sus efectos en falla cardiaca descompensada son inciertos.

En un ensayo de 100 pacientes hospitalizados por insuficiencia cardiaca descompensada, la adición de espironolactona, independientemente de la respuesta a los diuréticos de asa, se asoció con una resolución más rápida de los síntomas y una mejor función renal $[15,23]$. Un estudio más pequeño sugirió que los beneficios del antagonista del receptor de mineralocorticoides se mantienen incluso en pacientes con resistencia al diurético de asa [23].

Aunque los inhibidores de la anhidrasa carbónica, como la acetazolamida y otros diuréticos como los vaptanes (tolvaptán), pueden aumentar la diuresis en pacientes con SCR, la evidencia es escasa y no concluyente en cuanto a los desenlaces a nivel renal o cardiaco en pacientes con insuficiencia cardiaca descompensada [25-29]. Existe una necesidad imperiosa de encontrar nuevas intervenciones que mejoren las alternativas disponibles de manera segura y que mejoren el éxito de la descongestión en la insuficiencia cardiaca descompensada con sobrecarga hídrica. En curso se encuentra el estudio Advor, a completarse en el año 2022, el cual compara el efecto descongestivo de diurético de asa más el placebo vs. diurético de asa más acetazolamida [29].

Los inhibidores del cotransportador de sodio-glucosa 2 (SGLT2) tienen beneficios más allá de la reducción de la glucosa, por mecanismos que aún no están bien establecidos [30-32]. En el 2019 con el estudio DAPA-HF y recientemente con Emperor-Reduced se supone una nueva era en el tratamiento de la insuficiencia cardiaca con fracción de eyección reducida sin diabetes mellitus, estos estudios han mostrado una reducción del riesgo combinado de muerte de origen cardiovascular u hospitalización por descompensación de insuficiencia cardiaca [33,34]; sin embargo, el beneficio de estos medicamentos se limita a pacientes con insuficiencia cardiaca más avanzada pero estable, sin conocer realmente su desempeño en el paciente con sobrecarga hídrica y SCR [32,35]. Sin duda alguna, en el futuro cercano tendremos nuevos estudios que nos mostrarán su efecto en pacientes con estas características.

3. En tercer lugar, se propone la administración concomitante de solución salina hipertónica (SSH) como una terapia complementaria para los diuréticos de asa intravenosa, para mejorar o restaurar la eficacia farmacológica inicial [2,23]. Entre los mecanismos propuestos para explicar sus beneficios, se ha considerado que evitaría el agotamiento intravascular y, por lo tanto, mantendría el flujo sanguíneo renal y la tasa de filtración glomerular durante el tratamiento con furosemida intravenosa [2]. El estudio SMAC-HF se realizó en pacientes con insuficiencia cardiaca y resistencia diurética que fueron asignados al azar a SSH (150 ml) más furosemida IV de dosis alta dos veces al día (250 mg) y una restricción moderada de sodio $(120 \mathrm{mmol})$, en comparación con el mismo régimen 
diurético sin SSH y una dieta baja en sodio $(80 \mathrm{mmol})$ (36). Aquellos que recibieron SSH mostraron un aumento significativo en la diuresis y una menor estancia hospitalaria, con un efecto favorable sobre el aclaramiento de creatinina; durante el seguimiento, el grupo de SSH y restricción moderada de sodio tuvo menores tasas de reingreso y mortalidad [36].

En comparación con la administración de altas dosis de furosemida intravenosa sola, el uso concomitante de $\mathrm{SSH}$ ha demostrado una resolución clínica más rápida y completa de la congestión, ya que mejora el flujo renal al provocar expansión del volumen circulante eficaz con aumento de la presión hidrostática a nivel glomerular y peritubular, incrementándose la natriuresis y la concentración diurética, un potencial para proteger contra el deterioro de la función renal $[2,15]$.

A continuación, exponemos un algoritmo para el abordaje de la resistencia al diurético (figuras 1 y 2).
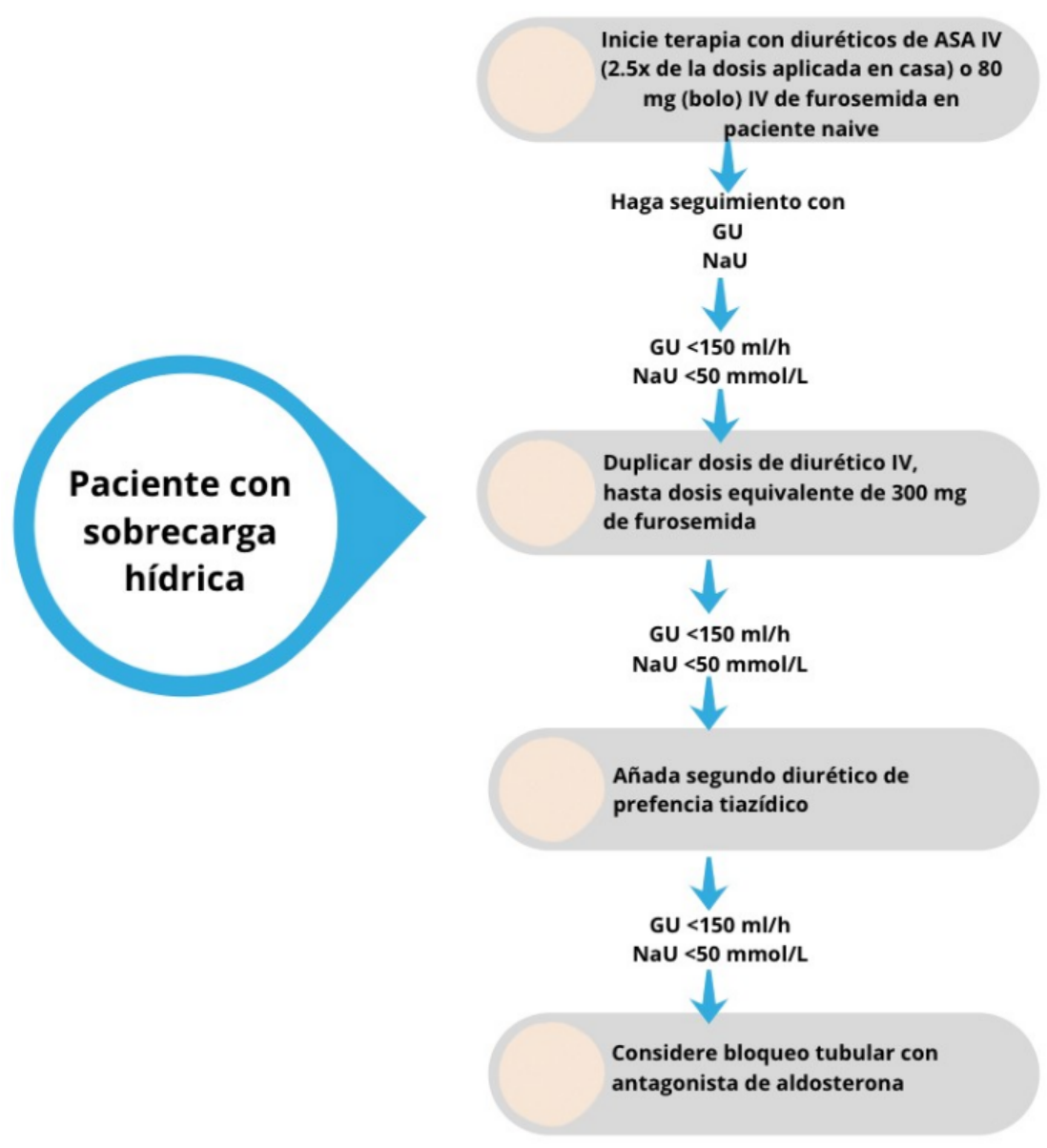

Figura 1. Abordaje propuesto para el paciente con sobrecarga hídrica

Fuente: elaboración con base en JACC, 75(10), 2020. 


\title{
Como manejar la resistencia al diurético:
}

\section{Optimice dosis de diurético de ASA}

\section{Considere bloqueo tubular}

\author{
Considerar estrategias \\ alternativas (Solución \\ salina hipertónica)
}

Figura 2. Manejo de la resistencia al diurético

Fuente: elaboración con base en Costanzo M, Ronco C, Abraham W, Agostoni P, Barasch J, Fonarow $\mathrm{G}$, et al., 2017 [4].

Se han propuesto varios predictores para la resistencia a los diuréticos. Una de las más estudiadas es la presencia de congestión venosa renal y la alta resistencia de la arteria renal, riesgo que se vuelve aún mayor cuando ambos factores están presentes conduciendo a la resistencia diurética [37].

Una estrategia para evaluar dichos parámetros es la ecografía Doppler de la hemodinámica arterial y venosa, que es una técnica no invasiva, de bajo costo y fácilmente disponible para la evaluación de los riñones y que permite valorar la anatomía, la perfusión y las presiones estimadas [38]. Se ha considerado que el principal determinante en la respuesta a los diuréticos en un paciente con insuficiencia cardiaca es la interrelación entre los parámetros hemodinámicos relacionados con el flujo sanguíneo renal, por lo que la ecografía Doppler se podría considerar un método confiable para la estratificación pronóstica de los pacientes con SCR [37]. Dentro de las mediciones potenciales de la Doppler renal, el índice de resistencia renal (IRR) es una medida del flujo sanguíneo renal que refleja las anomalías renales vasculares y parenquimatosas, por lo que representa una herramienta útil para detectar anomalías en la perfusión renal, que a su vez se asociaría a la progresión de la insuficiencia cardiaca [37,39]. Es 
calculada por el análisis espectral de la forma de onda Doppler arterial usando la fórmula de Pourcelot, es decir, la velocidad sistólica máxima menos la velocidad diastólica final, dividida por la velocidad sistólica máxima [38].

En adultos sanos, los valores normales de IRR son de alrededor de 0,60 , con una diferencia entre los dos riñones de menos del 5-8\%; un IRR de 0,70 o superior se considera anormal y generalmente predice un resultado desfavorable [38]. Ciccone, et al. en la universidad de Bari, en Italia, valoraron el IRR en pacientes con insuficiencia cardiaca que reciben diuréticos de asa, evidenciando que un IRR $\geq 0,71$ se asoció tanto a una dosis diurética basal significativamente más alta, lo que sugiere que desarrollarían resistencia diurética [39-41].

En condiciones normales, una disminución en el gasto cardiaco hará que el sistema autorregulador reduzca las resistencias renales para mantener el flujo renal dentro del rango normal; sin embargo, cuando hay alteración de estos sistemas reguladores por mecanismos neurohormonales, como sucede en la insuficiencia cardiaca, podría conllevar al aumento de las resistencias intrarrenales, lo que resultará en una filtración glomerular deteriorada [38,39].

Es importante tener en cuenta que existen otros factores que pueden alterar las resistencias renales como el aumento de la rigidez arterial, la aterosclerosis arterial, las lesiones parenquimatosas y la congestión venosa renal [37,38]. La congestión venosa renal puede conllevar al incremento de la resistencia de la arteria renal, sin embargo, también se ha considerado como un factor independiente del IRR, dado que incrementa la presión intersticial renal y la presión capilar glomerular, deteriorando la función de filtración [37].

El alto IRR y la congestión venosa renal, en el contexto de insuficiencia cardiaca, se han asociado independientemente con empeoramiento de la función renal y con el uso de altas dosis de diuréticos de asa, sobretodo si ambos factores están presentes, por lo que podrían predecir una respuesta diurética deficiente [37-39]. Una de las mayores desventajas para esta medida como predictor de resistencia al diurético es que al tratarse de una medición ecográfica, su validez será operador dependiente [42].

Se han propuesto otras medidas para prever la efectividad de la terapia descongestiva, según la resistencia al diurético como dispositivos de monitorización de presión venosa central, presión pulmonar, aun sin uso de manera generalizada en esta población [43].

Para intentar definir si la terapia al diurético es eficiente, se debe tener en cuenta la dosis del diurético utilizado, el gasto urinario, el cambio en el peso corporal y la excreción de sodio 
[44]. Algunos autores la definen como la atenuación del máximo efecto diurético que limita la excreción de sodio y de cloro; la resistencia a lo diurético se asocia con un empeoramiento en la función renal y un mayor riesgo de hospitalización por falla cardiaca y mortalidad [1].

\section{Terapias de reemplazo renal}

La ultrafiltración consiste en la extracción de agua plasmática a través de fibras huecas de una membrana semipermeable o hemofiltro, en respuesta a un gradiente de presión transmembrana. Se han desarrollado dispositivos simplificados que permiten manejar bajos flujos sanguíneos dentro de un sistema extracorpóreo con capacidad inferior a $50 \mathrm{~mL}$, con tasas de ultrafiltración entre 0 y $500 \mathrm{~mL}$ con accesos venosos centrales o periféricos, los cuales garanticen un flujo de sangre requerido, en muchos casos sin necesidad de trasladar al paciente a una unidad de cuidados intensivos $[42,45]$.

La ultrafiltración temprana puede ser una alternativa a los diuréticos intravenosos en pacientes con insuficiencia cardiaca descompensada con datos de sobrecarga hídrica, con beneficios potenciales que incluyen menor pérdida de potasio, menor liberación de renina y aldosterona y mayor pérdida de sodio $[1,46]$.

Unload fue un estudio prospectivo, aleatorizado y multicéntrico que comparó la ultrafiltración frente al tratamiento convencional con diuréticos endovenosos, encontrándose que la ultrafiltración produce de manera segura una mayor pérdida de peso y líquidos que los diuréticos intravenosos, con una reducción significativa en las tasas de rehospitalización a los 90 días; sin embargo, no queda claro si los resultados obtenidos podrían haberse logrado en el brazo diurético con una dosis más agresiva [43].

En un intento de valorar la eficacia y la seguridad de la ultrafiltración en pacientes con insuficiencia cardiaca descompensada, complicada por la congestión persistente y el deterioro de la función renal, se realizó el estudio Carress-HF, en el que se comparó una estrategia escalonada de terapia farmacológica con la ultrafiltración, evidenciándose que el tratamiento médico intensivo fue superior a la ultrafiltración para la preservación de la función renal a las 96 horas, con una pérdida de peso similar con los dos enfoques, teniendo en cuenta que la ultrafiltración se asoció con una tasa mayor de eventos adversos [47].

El estudio Avoid-HF, diseñado como un estudio aleatorizado y multicéntrico, comparó la ultrafiltración y el tratamiento médico en la insuficiencia cardiaca descompensada, con regímenes de tratamiento ajustables en lugar de fijos, en ambos brazos. El grupo de ultrafiltración tuvo una discreta tendencia, no significativa, a mayores días libres hasta el 
primer evento de descompensación de la falla cardiaca, con menos eventos cardiovasculares. Además, se evidenció que en el grupo de ultrafiltración se experimentaron mayores eventos adversos como sangrado, infección y trombosis venosa asociados al catéter [42].

La utilidad de la ultrafiltración es latente para pacientes con alta resistencia diurética y reingresos frecuentes por insuficiencia cardiaca descompensada, como una terapia para manejo de síntomas o de paliación para disminuir hospitalizaciones. En la actualidad, las guías recomiendan la UF para ese grupo selecto de pacientes. Resta además estandarizar los parámetros para programar la tasa de ultrafiltración ajustándose a las variables hemodinámicas y a la función renal de cada paciente, como lo hizo el estudio Unload [4].

\section{Diálisis peritoneal en falla cardiaca refractaria}

La diálisis peritoneal (DP) como técnica de UF fue descrita en 1949 por Schneierson y en los últimos años se ha utilizado como opción de tratamiento para pacientes con falla cardiaca refractaria; en comparación con la hemodiálisis, ofrece algunas ventajas como lo son una mayor preservación de la función renal residual y continuidad en la ultrafiltración con menos compromiso de la estabilidad hemodinámica $[48,49]$. Además, se han demostrado otros beneficios como depuración de moléculas proinflamatorias como la interleuquina 1 (IL-1) y factor de necrosis tumoral (TNF) que afectan la contractilidad miocárdica y se asocian con progresión de la falla cardiaca; también en aquellos pacientes con ascitis se reduce la presión intraabdominal, aliviando los síntomas derivados y mejorando la calidad de vida de los pacientes $[49,50]$.

Estudios realizados han demostrado la efectividad de la DP para ultrafiltración y para la mejoría de los síntomas congestivos, disminución de tasa y duración de hospitalizaciones por descompensación de falla cardiaca, sin que se evidencie deterioro en la función renal [48,51]. Debido a que las cohortes en los distintos estudios incluyen pocos pacientes, con muestras heterogéneas y gran carga de morbilidades, no se ha logrado establecer un beneficio con respecto a la sobrevida a los 12 meses, en comparación al tratamiento médico [48,49].

Las modalidades que se han empleado son: diálisis peritoneal intermitente, diálisis peritoneal continua ambulatoria (CAPD) y la diálisis peritoneal automatizada (APD) [48, 49].

Desde el punto de vista económico, en algunos trabajos se ha determinado una reducción de cuatro veces en costos anuales derivados de la atención hospitalaria en comparación con la terapia estándar $[48,49,51]$. Se resumen las ventajas y las desventajas para cada una de las alternativas de manejo en la figura 3. 


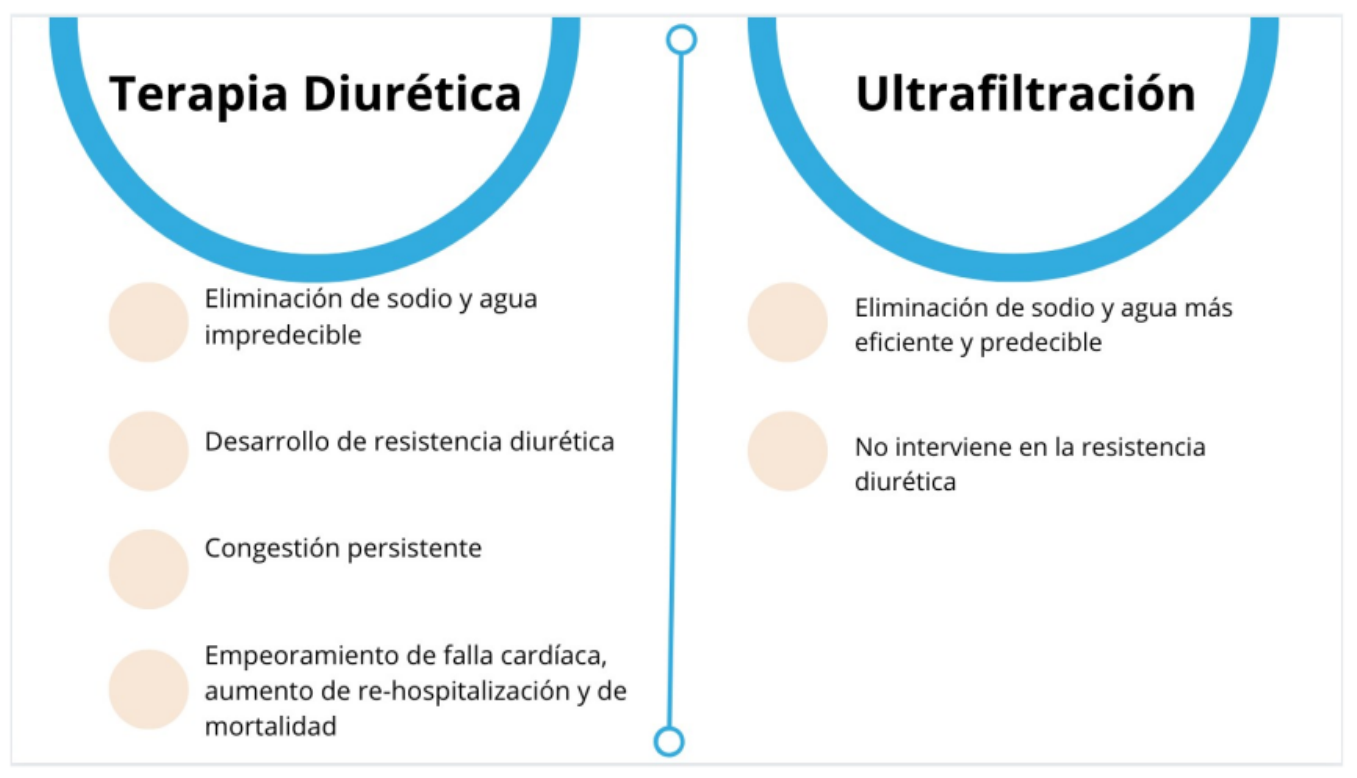

Figura 3. Ventajas y desventajas para las dos estrategias

Fuente: elaboración con base en Costanzo, et al. [4].

\section{Conclusión}

El SCR en sus diferentes presentaciones hace parte de una gran variedad de relaciones interdependientes entre el sistema cardiaco y el renal. La presencia de signos de congestión constituye uno de sus grandes desafíos, motivo por el cual se dan la mayoría de las hospitalizaciones en el caso de la insuficiencia cardiaca.

Como se ha descrito, a la luz de la terapéutica actual, la piedra angular del manejo son los diuréticos de asa, sin embargo, a medida que progresa la insuficiencia cardiaca y se utilizan de manera repetitiva los diuréticos, hay una respuesta inadecuada a estos. Los enfoques adicionales, como la optimización de las dosis del diurético de asa, el bloqueo tubular con agentes diuréticos tiazídicos o antagonistas de la aldosterona en dosis altas y el uso de SSH, son alternativas para contrarrestar la resistencia al diurético. Hay otras alternativas como los inhibidores de anhidrasa carbónica e iSGLT2 que pudieran ser opciones seguras que mejoren el éxito de la descongestión, sin embargo, aún no cuentan con estudios que soporten su uso en los pacientes con SCR.

La ultrafiltración y la DP son alternativas terapéuticas con potenciales ventajas dadas por la eliminación predecible del sodio corporal. Es necesario continuar el estudio para delimitar y dar manejo a la sobrecarga hídrica, la cual es la principal queja de los pacientes con insuficiencia cardiaca, además de la rehospitalización y la morbimortalidad de nuestros pacientes. 


\section{Consideraciones éticas}

Los autores declaran que los procedimientos seguidos se realizaron conforme a las normas éticas del comité de experimentación humana responsable y de acuerdo con lo establecido por la Asociación Médica Mundial en la Declaración de Helsinki; que han seguido los protocolos de su centro de trabajo sobre la publicación de datos de pacientes y que han obtenido el consentimiento informado de los pacientes o sujetos referidos en el artículo.

\section{Contribución de los autores}

María Carolina Manzur: conceptualización, investigación, escritura (manuscrito original) y escritura (revisión y edición); Ana Milena Mejía: conceptualización, investigación, validación, escritura (manuscrito original) y escritura (revisión y edición); Marco Anaya: conceptualización, investigación, validación, visualización, escritura (manuscrito original) y escritura (revisión y edición); Juan Camilo García: conceptualización, investigación, escritura (manuscrito original) y escritura (revisión y edición); Alejandra Molano: conceptualización, investigación, escritura (manuscrito original) y escritura (revisión y edición).

\section{Conflicto de intereses}

Los autores no declaran ningún conflicto de interés asociado al desarrollo de la investigación.

\section{Financiación}

Esta investigación no recibe ningún soporte financiero.

\section{Referencias}

[1] Rangaswami J, Bhalla V, Blair J, Chang T, Costa S, Lentine K, et al. Cardiorenal syndrome: classification, pathophysiology, diagnosis, and treatment strategies: A scientific statement from the American Heart Association. Circulation. 2019 abr. 16;139(16):e840-78. https://doi. org/10.1161/CIR.0000000000000664 个Ver páginas 1, 2, 3, 4, 5, 6, 11

[2] Bielecka-Dabrowa A, Godoy B, Schefold J, Koziolek M, Banach M, Bielecka- dabrowa A. Decompensated heart failure and renal failure : What is the current evidence? Curr Heart Fail Rep. 2018;15(4):224-38. https://doi.org/10.1007/s11897-018-0397-5 个Ver páginas 1, 2, 3, 7, 8 
[3] Dizeo C, Navarta DA, Franchi VM, Álvarez S, Forcinito D, Trejo G, et al. Síndrome cardiorrenal como predictor de mala evolución intrahospitalaria en pacientes añosos internados con insuficiencia cardíaca. Insufic Card. 2012;7(3):102-8. $\uparrow$ Ver página 2

[4] Costanzo M, Ronco C, Abraham W, Agostoni P, Barasch J, Fonarow G, et al. Extracorporeal ultrafiltration for fluid overload in heart failure. J Am Cardiol. 2017;69(19):2428-45. https:/ doi.org/10.1016/j.jacc.2017.03.528 个Ver páginas 2, 9, 12, 13

[5] Yogasundaram H, Chappell MC, Braam B, Oudit GY. Cardiorenal syndrome and heart failure d challenges and opportunities. Can J Cardiol [Internet]. 2019;35(9):1208-19. https:/ doi.org/10.1016/j.cjca.2019.04.002 个Ver páginas 3, 4, 5

[6] Boerrigter G, Burnett JC. Cardiorenal syndrome in decompensated heart failure: prognostic and therapeutic implications. Curr Heart Fail Rep. 2004;1(3):113-20. https://doi.org/ 10.1007/s11897-004-0020-9 个Ver página 3

[7] Brosius FC, Hostetter TH, Kelepouris E, Mitsnefes MM, Moe SM, Moore MA, et al. Detection of chronic kidney disease in patients with or at increased risk of cardiovascular disease: A science advisory from the American Heart Association Kidney and Cardiovascular Disease Council; the Councils on High Blood Pressure Research, Cardiovascular Disease in the Young, and Epidemiology and Prevention; and the Quality of Care and Outcomes Research Interdisciplinary Working Group. Circulation. 2006;114(10):10837. https://doi.org/10.1161/CIRCULATIONAHA.106.177321 个Ver página 3

[8] Sarnak MJ, Levey AS, Schoolwerth AC, Coresh J, Culleton B, Hamm LL, et al. Kidney disease as a risk factor for development of cardiovascular disease: A statement from the American Heart Association Councils on Kidney in Cardiovascular Disease, High Blood Pressure Research, Clinical Cardiology, and Epidemiology and Prevention. Hypertension.

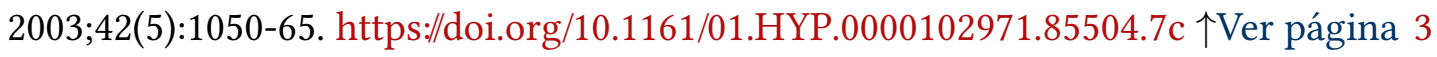

[9] Mahon NG, Blackstone EH, Francis GS, Starling RC, Young JB, Lauer MS. The prognostic value of estimated creatinine clearance alongside functional capacity in ambulatory patients with chronic congestive heart failure. J Am Coll Cardiol [Internet]. 2002;40(6):110613. https://doi.org/10.1016/S0735-1097(02)02125-3 个Ver página 3

[10] Agrawal A, Naranjo M, Kanjanahattakij N, Rangaswami J, Gupta S. Cardiorenal syndrome in heart failure with preserved ejection fraction - an under-recognized clinical entity. Heart Fail Rev. 2019;24:421-37. https://doi.org/10.1007/s10741-018-09768-9 $\uparrow$ Ver páginas 3, 4

[11] Otukesh H, Mehralizadeh S. Cardiorenal syndrome or renocardial syndrome: A review. J Pediatr Nephrol. 2016;4(1):14-24. https://doi.org/10.4061/2011/762590 个Ver página 3 
[12] McCullough PA. Cardiorenal syndromes: Pathophysiology to prevention. Int J Nephrol. 2011;2011:1-10. https://doi.org/10.4061/2011/762590 个Ver página 3

[13] Cruz DN. Cardiorenal syndrome in critical care: The acute cardiorenal and renocardiac syndromes. Adv Chronic Kidney Dis [Internet]. 2013;20(1):56-66. https:/doi.org/10.1053/j. ackd.2012.10.005 个Ver página 3

[14] Thind G, Loehrke M, Wilt JL. Acute cardiorenal syndrome: Mechanisms and clinical implications. Cleve Clin J Med. 2018;85(3):231-9. https:/doi.org/10.3949/ccjm.85a.17019 个Ver página 3

[15] Villanueva L, Sánchez B, Sanjines D. Tratamiento congestivo : diuréticos, resistencia diurética y alternativas. Papel de la ultrafiltración. Insufic Card. 2018;13(2):72-86. $\uparrow$ Ver páginas $3,6,7,8$

[16] Berl T, Henrich W. Kidney-heart interactions: epidemiology, pathogenesis, and treatment. Clin J Am Soc Nephrol. 2006;1(1):8-18. https://doi.org/10.2215/CJN.00730805 个Ver página 4

[17] Pereira-Rodríguez JE, Boada-Morales L, Niño-Serrato DR, Caballero-Chavarro M, Rincón-Gonzales G, Jaimes-Martin T, et al. Síndrome cardiorrenal. Rev Colomb Cardiol. 2017;24(6):602-13. https://doi.org/10.1016/j.rccar.2017.05.019 个Ver página 5

[18] Hatamizadeh P, Fonarow GC, Budoff MJ, Darabian S, Kovesdy CP, Kalantar- Zadeh K. Cardiorenal syndrome: Pathophysiology and potential targets for clinical management. Nat Rev Nephrol [Internet]. 2013;9(2):99-111. https://doi.org/10.1038/nrneph.2012.279 个Ver página 5

[19] Chitturi C, Novak JE. Diuretics in the management of cardiorenal syndrome. Adv Chronic Kidney Dis [Internet]. 2018;25(5):425-33. https:/doi.org/10.1053/j.ackd.2018.08.008 $\uparrow$ Ver páginas 5,6

[20] Mullens W, Damman K, Testani JM, Martens P, Mueller C, Lassus J, et al. Evaluation of kidney function throughout the heart failure trajectory - a position statement from the Heart Failure Association of the European Society of Cardiology. Eur Soc Cardiol. 2020;22(4). https:/doi.org/10.1002/ejhf.1697 个Ver página 5

[21] Boorsma EM, Maaten JM, Damman K, Dinh W, Gustafsson F, Goldsmith S, et al. Congestion in heart failure: A contemporary look at physiology, diagnosis and treatment. Nat Rev Cardiol [Internet]. 2020 my. 15. https://doi.org/10.1038/s41569-020-0379-7 $\uparrow$ Ver página 5 
[22] Felker GM, Lee KL, Bull DA, Redfield MM, Stevenson LW, Goldsmith SR, et al. Diuretic strategies in patients with acute decompensated heart failure. N Engl J Med. 2011;364(9):797805. https://doi.org/10.1056/NEJMoa1005419 ^Ver páginas 5, 6

[23] Bowman B, Nawarskas J, Anderson J. Treating diuretic resistance. Cardiol Rev. 2016;24(5):256-60. https://doi.org/10.1097/CRD.0000000000000116 个Ver páginas 6, 7

[24] De-Bruyne L. Mechanisms and management of diuretic resistance in congestive heart failure. Postgrad Med J. 2003;79:268-72. https://doi.org/10.1136/pmj.79.931.268 个Ver página 6

[25] Jentzer JC, Bihorac A, Brusca SB, Del-Rio-Pertuz G, Kashani K, Kazory A, et al. Contemporary management of severe acute kidney injury and refractory cardiorenal syndrome: JACC Council perspectives. J Am Coll Cardiol. 2020;76(9):1084-101. https://doi.org/10.1016/ j.jacc.2020.06.070 个Ver página 7

[26] Cox ZL, Hung R, Lenihan DJ, Testani JM. Diuretic strategies for loop diuretic resistance in acute heart failure: The 3T trial. JACC Hear Fail. 2020;8(3):157- 68. https://doi.org/10.1016/ j.jchf.2019.09.012 个Ver página 7

[27] Gupta R, Testani J, Collins S. Diuretic resistance in heart failure. Curr Hear Fail Rep. 2019;16(2):57-66. https://doi.org/10.1007/s11897-019-0424-1 个Ver página 7

[28] Greene SJ, Felker GM, Giczewska A, Kalogeropoulos AP, Ambrosy AP, Chakraborty H, et al. Spironolactone in acute heart failure patients with renal dysfunction and risk factors for diuretic resistance: From the ATHENA-HF Trial. Can J Cardiol [Internet]. 2019;35(9):1097105. https://doi.org/10.1016/j.cjca.2019.01.022 个Ver página 7

[29] Mullens W, Verbrugge FH, Nijst P, Martens P, Tartaglia K, Theunissen E, et al. Rationale and design of the ADVOR (Acetazolamide in Decompensated Heart Failure with Volume Overload) trial. Eur J Heart Fail. 2018;20(11):1591-600. https://doi.org/10.1002/ejhf.1307 个Ver página 7

[30] Kato ET, Kimura T. Sodium-glucose co-transporters-2 inhibitors and heart failure: State of the art review and future potentials. Int J Hear Fail. 2020;2(1):12. https://doi.org/10.36628/ ijhf.2019.0013 $\uparrow$ Ver página 7

[31] Lam C, Chandramouli C, Ahooja V, Verma S. SGLT-2 inhibitors in heart failure: Current management, unmet needs, and therapeutic prospects. J Am Heart Assoc. 2019;8(20):1-12. https://doi.org/10.1161/JAHA.119.013389 个Ver página 7

[32] Zannad F, Ferreira JP, Pocock SJ, Anker SD, Butler J, Filippatos G, et al. SGLT2 inhibitors in patients with heart failure with reduced ejection fraction: A meta-analysis of 
the EMPEROR-Reduced and DAPA-HF trials. Lancet. 2020;396(10254):819-29. https://doi.org/ 10.1016/S0140-6736(20)31824-9 个Ver página 7

[33] Packer M, Anker SD, Butler J, Filippatos G, Pocock SJ, Carson P, et al. Cardiovascular and renal outcomes with empagliflozin in heart failure. N Engl J Med. 2020;383(15):1413-24. https://doi.org/10.1056/NEJMoa2022190 个Ver página 7

[34] McMurray J, Solomon S, Inzucchi S, Køber L, Kosiborod M, Martinez FA, et al. Dapagliflozin in patients with heart failure and reduced ejection fraction. N Engl J Med. 2019;381(21):1995-2008. https://doi.org/10.1056/NEJMoa1911303 个Ver página 7

[35] Zannad F, Rossignol P. Cardiorenal syndrome revisited. Circulation. 2018;138:929-44. https://doi.org/10.1161/CIRCULATIONAHA.117.028814 $\uparrow$ Ver página 7

[36] Paterna S, Fasullo S, Parrinello G, Cannizzaro S, Basile I. Short-term effects of hypertonic saline solution in acute heart failure and long-term effects of a moderate sodium restriction in patients with compensated heart failure with New York Heart Association Class III (Class C) (SMAC-HF Study). Am J Med Sci [Internet]. 2011;342(1):27-37. https://doi.org/10.1097/ MAJ.0b013e31820f10ad $\uparrow$ Ver página 8

[37] Iacoviello $M$, Leone $M$. The renal arterial resistance index predicts worsening renal function in chronic heart failure patients. Cardiorenal Med. 2017;7:42-9. https://doi.org/10.1159/ $000448405 \uparrow$ Ver páginas 9, 10

[38] Grande D, Terlizzese P, Iacoviello M. Role of imaging in the evaluation of renal dysfunction in heart failure patients. World J Nephrol. 2017;6(3):86-167. https://doi.org/10.5527/ wjn.v6.i3.123 $\uparrow$ Ver páginas 9, 10

[39] Doronzo A, Iacoviello M, Paradies V, Antoncecchi V, Monitillo F, Citarelli G, et al. Presence of high renal arterial resistance index is independently associated with the increase of loop diuretic diuretic dose in heart failure outpatients. Eur Heart J. 2014;35. https://doi.org/ 10.1093/eurheartj/eht307.P636 个Ver páginas 9, 10

[40] Iacoviello M, Doronzo A, Paradies V, Antoncecchi V, Monitillo F, Citarelli G, et al. The independent association between altered renal arterial resistance and loop diuretic dose in chronic heart failure outpatients. IJC Hear Vasc [Internet]. 2015;7:119-23. https://doi.org/ 10.1016/j.ijcha.2015.03.004 个Ver página 10

[41] Ciccone MM, Iacoviello M, Gesualdo L, Puzzovivo A, Antoncecchi V, Doronzo A, et al. The renal arterial resistance index: A marker of renal function with an independent and 
incremental role in predicting heart failure progression. Eur J Heart Fail. 2014;16:210-6. https://doi.org/10.1002/ejhf.34 个Ver página 10

[42] Costanzo MR, Negoianu D, Jaski BE, Bart BA, Heywood JT, Anand IS, et al. Aquapheresis versus intravenous diuretics and hospitalizations for heart failure. JACC Hear Fail. 2016;4(2):95-105. https://doi.org/10.1016/j.jchf.2015.08.005 个Ver páginas 10, 11, 12

[43] Costanzo MR, Guglin ME, Saltzberg MT, Jessup ML, Bart BA, Teerlink JR, et al. Ultrafiltration versus intravenous diuretics for patients hospitalized for acute decompensated heart failure. J Am Coll Cardiol. 2007;49(6):675-83. https:/doi.org/10.1016/j.jacc.2006.07.073 个Ver página 10,11

[44] Felker GM, Ellison DH, Mullens W, Cox ZL, Testani JM. Diuretic therapy for patients with heart failure. J Am Coll Cardiol. 2020;75(10):1178-95. https:/doi.org/10.1016/j.jacc.2019. 12.059 个Ver página 11

[45] Rimondini A, Cipolla CM, Della-Bella P, Grazi S, Sisillo E, Susini G, et al. Hemofiltration as short-term treatment for refractory congestive heart failure. Am J Med. 1987;83(1):43-8. https://doi.org/10.1016/0002-9343(87)90495-5 个Ver página 11

[46] Agostoni PG, Marenzi GC. Sustained benefit from Ultrafiltration in moderate congestive heart failure. Cardiology. 2001;96(3-4):183-9. https://doi.org/10.1159/000047402 $\uparrow$ Ver página 11

[47] Bart B, Goldsmith S, Lee K, Givertz M, O’Connor C, Bull D, et al. Ultrafiltration in decompensated heart failure with cardiorenal syndrome. N Engl J Med. 2012;367(24):2296-304. https:/doi.org/10.1056/NEJMoa1210357 个Ver página 11

[48] Grossekettler L, Schmack B, Meyer K, Brockmann C, Wanninger R, Kreusser MM, et al. Peritoneal dialysis as therapeutic option in heart failure patients. ESC Hear Fail. 2019;6:2719. https://oi.org/10.1002/ehf2.12411 个Ver páginas 12

[49] Montejo J, Bajo M, Peso G, Selgas R. Papel de la diálisis peritoneal en el tratamiento de la insuficiencia cardíaca refractaria. Rev Nefrol. 2010;30:21-7. 个Ver páginas 12

[50] Kazory A. Peritoneal dialysis for chronic cardiorenal syndrome: Lessons learned from ultrafiltration trials. World J Cardiol. 2015;7(7):392-7. https:/doi.org/10.4330/wjc.v7.i7.392 $\uparrow$ Ver página 12

[51] Viglino G, Neri L, Feola M. Peritoneal ultrafiltration in congestive heart failure - findings reported from its application in clinical practice: A systematic review. Am J Nephrol. 2015;28:29-38. https://doi.org/10.1007/s40620-014-0166-9 个Ver páginas 12 